\title{
Remarks on the Meaning of Analogical Relations
}

\author{
Ulf Krumnack ${ }^{1}$ and Helmar Gust ${ }^{1}$ and Angela Schwering ${ }^{2}$ and Kai-Uwe Kühnberger ${ }^{1}$ \\ ${ }^{1}$ Institute of Cognitive Science \\ University of Osnabrück, Germany \\ \{krumnack, hgust, kkuehnbe\}@uos.de \\ ${ }^{2}$ Institute of Geoinformatics \\ University of Münster \\ schwering@uni-muenster.de
}

\begin{abstract}
Analogical reasoning plays an important role in the context of higher cognitive abilities of humans. Analogies can be used not only to explain reasoning abilities of humans, but also to explain learning from sparse data, creative problem solving, abstractions of concrete situations, and recognition of formerly unseen situations, just to mention some examples. Research in AI and cognitive science has been proposing several different models of analogy making. Nevertheless, no approach for a model theoretic semantics of analogy making is currently available. This paper gives an analysis of the meaning (the semantics) of analogical relations that are computed by the analogy engine HDTP (Heuristic-Driven Theory Projection).
\end{abstract}

\section{Introduction}

Humans show remarkable higher cognitive abilities comprising not only (abstract) types of reasoning, learning from sparse data, and planning, but also the ability to creatively finding new conceptualizations of an unknown domain, to solve problems based on similar solutions for other problems, and to recognize and categorize perceptual input that was never seen before. At least to a certain extent it is possible to explain and to model such types of higher cognitive abilities with frameworks for analogical reasoning (GHK01).

Because of the wide range of applicability of analogy making mechanisms for the explanation of various higher cognitive abilities, analogies are of interest not only for artificial intelligence and cognitive science, but also for artificial general intelligence. Classical AI methodologies suffer from various deficiencies that result from highly specialized models. For example, it is hard to find uniform frameworks for the variety of different reasoning types (GKSK09), for creativity aspects of human cognition (Ind92), or for problem solving in unknown situations (GP07). As a consequence of these deficiencies the desired generalization capabilities of appropriate AGI systems are currently far from being reachable. To tackle this problem we propose the usage of analogy mechanisms for AGI systems.

Frameworks for analogy making cover a large part of cognitive abilities that are usually considered to be central for AGI systems. A good source to support this claim is (KHG09) where analogies are used to model aspects of reasoning, creativity, problem solving, learning, perception, or motor control, just to mention some of them. Furthermore, even from a more abstract perspective the establishment of analogical relations seems to be one of the rare possibilities to explain many cognitive phenomena in a uniform way: quite often we act (alternatively perceive, reason, learn etc.) as if we were in another (well-known and analogous) situation. It rarely happens that humans can reason in a purely deductive (abductive, inductive etc.) way to act in real life. A natural description of such cognitive phenomena can be provided by analogies, because vagueness, learning, and the transfer of knowledge about old situations to new ones are intrinsically embedded in the very idea of analogy making.

Due to the fact that analogies are considered to be a central mechanism of human cognition and intelligence, a number of models have be proposed to explain different aspects of analogies, varying in complexity and in their degree of formalization. A few examples of such frameworks are SME (FFG89), interactionism (DIS03), LISA (HH96), Copycat (Hof95), and AMBR / DUAL (KP01). ${ }^{1}$ Most of these analogy making models use special means for representation and computation of analogical inferences. For example, the structure mapping engine (FFG89), probably the currently best known analogy model, uses a graph-based representation and a heuristic-governed matching algorithm.

Although, we can conclude that there is a broad variety of different computational models, it is hard to find a spelled out semantics of analogical relations computed by algorithmic models. Even worse, for all established frameworks even an endeavor to find a semantics of the underlying computations cannot be found. However, from an AGI perspective, it would be desirable to have a model that could be combined with standard mechanisms of knowledge representation and reasoning.

\footnotetext{
${ }^{1} \mathrm{~A}$ good overview of various theoretical and practical approaches for the modeling of analogy making can be furthermore found in the special issue of Cognitive Systems Research about analogical reasoning as a means for the integration of cognitive abilities (SKK09).
} 
In this paper, we will discuss heuristic-driven theory projection (HDTP), a formal framework to compute analogies between domains that are described by classical first-order formulas (GKS06). HDTP's matching algorithm is based on the syntactic representation and analogies are computed as mappings of formula sets induced by their domain axiomatizations (SKKG09). This approach fits into the classical paradigm of symbol processing, where operations are performed on a symbolic level in a way that a coherent semantic interpretation can be provided. However, from this perspective, the framework remains incomplete, until a semantic characterization of the syntactic operations is provided.

In this paper, we will show, that the syntactic mapping procedure of HDTP can be given a sensible interpretation on the semantic side. Not only, the syntactic mapping can be shown to induce a mapping between models (this was already shown in (GKKS07)), but furthermore, the generalized formulas constructed by HDTP during the mapping process can be interpreted as a new, abstract domain theory, which can be given a model theoretic semantics. To our knowledge this is the first concrete approach to make the semantics of analogy making formally precise.

The paper has the following structure. First, we will sketch the syntactic basis of HDTP and the challenges of developing a semantics for HDTP, then we will sketch the theory of institutions. We will continue with describing an institution theoretic analysis of analogy making. Finally, we will sketch an example and we will add some concluding remarks.

\section{Heuristic-Driven Theory Projection}

\section{The Theory in a Nutshell}

HDTP is a framework for discovering analogous structures of pairs of logical theories. In this section, we sketch the main ideas of the syntactic anti-unification process of HDTP. For a thorough introduction to the syntactic principles of HDTP, the reader is referred to (SKKG09).

- Two sets of formulas $A x_{S}$ and $A x_{T}$ are provided as input specifying an axiomatization of the source and target domain, respectively. Each axiomatization describes (some aspects) of a domain of knowledge. These sets of formulas $A x_{S}$ and $A x_{T}$ induce corresponding theories $T h_{S}$ and $T h_{T}$ (i.e. the deductive closure of $A x_{S}$ and $\left.A x_{T}\right)$.

- A pair of clauses $c_{S} \in T h_{S}$ and $c_{T} \in T h_{T}$ is selected and (syntactically) generalized by anti-unification to a clause $c_{G} \cdot{ }^{2}$ For generalization, domain symbols

\footnotetext{
${ }^{2}$ Anti-unification can be understood as the dual construction of unification, i.e. instead of computing the most general unifier, the most specific (least general) generalization of terms and formulas is computed. Anti-unification was introduced by Plotkin (Plo70).
}

- can be kept, if they occur in both domains (e.g. a relation symbol $>$ )

- can be generalized into a new variable, e.g. sun, nucleus are generalized to an individual variable $X$ in the famous Rutherford analogy describing the analogy between the solar system and the atom model

- can be dropped by an "argument insertion" substitution, i.e. symbols are "integrated" into a complex function.

As a by-product, the computed generalization provides a pair of substitutions $\langle\sigma, \tau\rangle$ with $c_{G} \sigma=c_{S}$ and $c_{G} \tau=c_{T}$. In other words, the source and target clauses $c_{s}$ and $c_{T}$ can be gained by applying the substitutions to the corresponding generalized clause $c_{G}$, respectively.

- A set $A x_{G}$ of generalized clauses is incrementally built by repeating the described generalization step. This set is considered to be good, if it has a low substitution complexity and a high degree of coverage ${ }^{3}$

- The substitutions belonging to a (good) set of generalizations can be used to establish an analogical relation

- Based on this relation, formulas of the source domain that are not anti-unified yet can be transferred (projected) to the target domain in order to allow new conceptualizations of the target.

\section{Challenges}

There are several challenges of this approach if one wants to develop a model theoretic semantics for HDTP. First, the generalized expression $A x_{G}$ are not necessarily first-order logical formulas (FOL formulas). The anti-unification process may result in a second-order generalization, for example, by introducing a relation or function variable. Second, the variables introduced by anti-unification might be treated differently than ordinary (universally quantified) variables. In particular, second-order variables can be interpreted as existentially quantified in order to prevent inconsistencies or they need to get a treatment as described below. Third, the question arises how an adequate notion for a "model" (in the logical model theoretic sense) of the resulting analogy might look like.

Here are the basic ideas of the present approach: In (GKS06), it is proposed, that the generalized terms can be seen as axioms of a generalized theory. A framework is sketched that integrates a semantics for the analogical relation and the generalized theory. However, some questions are left open and especially the status of the variables introduced by anti-unification remains

\footnotetext{
${ }^{3}$ Coverage is the concept that "measures" the degree of specificity of a generalization. Intuitively, we can say that an anti-unifier $\left\langle A x_{G}, \sigma, \tau\right\rangle$ has at least the same coverage as the anti-unifier $\left\langle A x_{G}^{\prime}, \sigma^{\prime}, \tau^{\prime}\right\rangle$ if there exists a substitution $\Theta: A x_{G}^{\prime} \rightarrow A x_{G}$, such that $\sigma^{\prime}=\sigma \circ \Theta$ and $\tau^{\prime}=\tau \circ \Theta$. Compare (SKKG09) for the details of this concept.
} 
unclear. If these generalized symbols would be considered as logical variables, the resulting formulas leave the realm of first-order logic, as anti-unification can also generalize function and predicate symbols. Therefore, we propose to see the generalized symbols not as variables, but as elements of a new vocabulary. The generalized theory is then a classical first-order theory with formulas built from these symbols. The substitutions induce a mapping between the generalized theory and the domain theories.

\section{An Institutional View on Analogies}

To make these ideas more precise, we will use the language of the theory of institutions (GB92). ${ }^{4}$ Institutions provide a framework to describe model theory at an abstract level using methods from category theory. Informally, an institution consists of a collection of signatures Sign and to each signature $\Sigma$, first the collection $\operatorname{Sen}(\Sigma)$ of all $\Sigma$-sentences is assigned. In the case of FOL, the $\Sigma$-sentences correspond to the set of all FOL formulas that can be built using symbols from $\Sigma$. Second, for each signature $\Sigma$ the collection $\operatorname{Mod}(\Sigma)$ of all $\Sigma$-models is assigned. In the case of FOL, this collection correspond to all possible interpretations of symbols from $\Sigma$. The $\Sigma$-models and $\Sigma$-sentences are related by the relation of $\Sigma$-satisfaction, usually denoted by $\models_{\Sigma}$.

A central idea of the theory of institutions is, that truth is invariant under the change of notation. Within an institution, signatures can be mapped to other signatures by so called signature morphisms, i.e. structure preserving functions. In FOL, this translates into the constraint that arity and sortal restrictions have to match. A signature morphism $f: \Sigma \rightarrow \Sigma^{\prime}$ induces functions $\operatorname{Sen}(f): \operatorname{Sen}(\Sigma) \rightarrow \operatorname{Sen}\left(\Sigma^{\prime}\right)$ and $\operatorname{Mod}(f):$ $\operatorname{Mod}\left(\Sigma^{\prime}\right) \rightarrow \operatorname{Mod}(\Sigma)$ which have to be consistent with $\Sigma$-satisfaction as specified by the following contravariant satisfaction constraint: for all $\varphi \in \operatorname{Sen}(\Sigma)$ and $M^{\prime} \in \operatorname{Mod}\left(\Sigma^{\prime}\right)$ :

$$
M^{\prime} \models_{\Sigma} \operatorname{Sen}(f)(\varphi) \Leftrightarrow \operatorname{Mod}(f)\left(M^{\prime}\right) \models_{\Sigma} \varphi
$$

Every collection $T$ of $\Sigma$-sentences (called $\Sigma$-theory) determines a class of $\Sigma$-models:

$$
T^{*}=\left\{M \mid M \models_{\Sigma} \varphi \text { for all } \varphi \in T\right\}
$$

Dually, every collection $\mathcal{V}$ of $\Sigma$-models determines a $\Sigma$-theory:

$$
\mathcal{V}^{*}=\left\{\varphi \mid M \models_{\Sigma} \varphi \text { for all } M \in \mathcal{V}\right\}
$$

As a natural consequence we get that the double application of these operators provides the closure under semantic entailment: $T^{\bullet}=T^{* *}$.

\footnotetext{
${ }^{4}$ For a thorough discussion of logic systems in the language of the theory of institutions the reader is referred to the monograph (Dia08).
}

\section{The Generalized Theory}

We now describe HDTP within the framework of institutions, more precisely, within the institution FOL. The new variables introduced by the process of antiunification are placeholders, which are instantiated by different terms on the source and target side. In what follows, we will treat these variables as new symbols which can be used as a signature for a generalized theory of both domains. Using this idea we can give the intuitive notion of "generalized theory" a formal meaning.

The signature $\Sigma_{G}$ will consist of all symbols used in the generalized formulas $A x_{G}$, i.e. symbols from $\Sigma_{S} \cap$ $\Sigma_{T}$ and the variables introduced by anti-unification. The generalized formulas $A x_{G}$ are then ordinary firstorder formulas over the signature $\Sigma_{G}$ in the usual sense. The theory spanned by these formulas will be called (as above) the generalized theory $T h_{\Sigma_{G}}\left(A x_{G}\right) .^{5}$

The generalized axioms $A x_{G}$ are related to the axiomatizations $A x_{S}$ and $A x_{T}$ of the source and target domain by the the following relations

$$
A x_{G} \sigma \subseteq\left(A x_{S}\right)^{\bullet} \quad \text { and } \quad A x_{G} \tau \subseteq\left(A x_{\tau}\right)^{\bullet}
$$

with $\sigma$ and $\tau$ being the substitutions computed by HDTP. The application of the substitutions $\sigma$ and $\tau$ defines mappings $\sigma^{*}: \operatorname{Sen}\left(\Sigma_{G}\right) \rightarrow \operatorname{Sen}\left(\Sigma_{S}\right)$ and $\tau^{*}: \operatorname{Sen}\left(\Sigma_{G}\right) \rightarrow \operatorname{Sen}\left(\Sigma_{T}\right)$. It can be shown that these mappings can be restricted to the respective theories, i.e. that $\sigma^{*} \mid:\left(A x_{G}\right)^{\bullet} \rightarrow\left(A x_{S}\right)^{\bullet}$ and $\tau^{*} \mid:\left(A x_{G}\right)^{\bullet} \rightarrow$ $\left(A x_{T}\right)^{\bullet}$. However, in general the induced mappings are not theory morphisms in the sense of (GB92), as there are no underlying signature morphisms. To overcome this problem, we will extend the original domain signatures $\Sigma_{S}$ and $\Sigma_{T}$.

\section{Substitutions}

In the setting of HDTP, there exists a set of (higherorder) generalized formulas, which can be instantiated via substitution to domain formulas over given signatures. We will describe this situation in terms of signatures and signature morphisms.

Let $A x$ be a set of (higher-order) formulas and $\theta$ be a substitution such that $A x \theta$ is a set of (first-order) formulas over some signature $\Sigma$. Now we can define a signature $\Sigma_{\theta}$ which allows to interpret the formulas $A x$ as first-order formulas as follows:

(i) We extend $\Sigma$ by taking into account the pairs $X / t$ in $\theta$ and by introducing a new symbol $X$ for each such pair. Notice, that there is an obvious embed$\operatorname{ding} \Sigma \hookrightarrow \Sigma_{\theta}$.

(ii) A system of equations and equivalences is used to relate new symbols in $\Sigma_{\theta}$ to those in $\Sigma$.

\footnotetext{
${ }^{5}$ From now on we will use $T h_{\Sigma}(A x)$ to denote the closure under semantic entailment of an axiomatization $A x$ restricted to sentences over a signature $\Sigma$, i.e. $T h_{\Sigma}(A x)=$ $(A x)^{\bullet} \cap \operatorname{Sen}(\Sigma)$.
} 
Consider again a substitution $\theta$ as above. We can define a set of $\Sigma_{\theta}$-formulas $D e f_{\theta}$ as follows: ${ }^{6}$

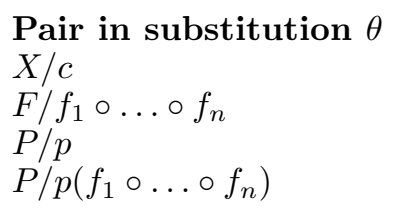

$$
\begin{aligned}
& \text { Formula in } \text { Def }_{\theta} \\
& X=X \theta \\
& \forall \bar{x} F(\bar{x})=F \theta(\bar{x}) \\
& P \leftrightarrow p \\
& \forall \bar{x} P(\bar{x}) \leftrightarrow P \theta(\bar{x})
\end{aligned}
$$

The idea is, that $D e f_{\theta}$ provides a description that allows the application of substitutions as a logical deduction in the HDTP algorithm. One can make this more precise by noticing the following fact: If $A x, \theta, \Sigma$ are given as above, then the following two facts hold:

- $T h_{\Sigma_{\theta}}\left(A x \cup D e f_{\theta}\right) \cap \operatorname{Sen}(\Sigma)=T h_{\Sigma}(A x \theta)$.

- There is a (natural) isomorphism

$$
\operatorname{Mod}_{\Sigma_{\theta}}\left(A x \cup D e f_{\theta}\right) \stackrel{\cong}{\longrightarrow} \operatorname{Mod}_{\Sigma}(A x \theta) \text {. }
$$

The crucial point is, that the additional newly introduced symbols do not allow to derive new sentences in $\operatorname{Sen}(\Sigma)$. Notice that they cannot introduce interrelations between existing symbols provided that these additional symbols are really new. On the semantic level, the additional symbols do not provide new models, as the interpretations of these symbols are fully constrained by the formulas from $D e f_{\theta}$.

\section{Coverage}

Usually, an analogy does not establish a complete mapping between two domains. Quite often only parts of the domains are associated with each other. This fact can be used to motivate the concept of coverage in HDTP. A domain formula $\varphi$ from $\operatorname{Sen}(\Sigma)$ is said to be covered by an analogy iff $\varphi \in \operatorname{Th}\left(A x_{G} \theta\right)$, i.e. $\phi$ is either an instantiation of a generalized formula or can be derived from such instantiations. The set $\operatorname{Th}\left(A x_{G} \theta\right)$ is called the part of the domain that is covered by the generalization (or analogy).

Assume that $A x_{D}$ is a set of domain axioms over some signature $\Sigma, A x_{G}$ a set of generalized formulas, $\theta$ a substitution that instantiates $A x_{G}$ in $\operatorname{Sen}(\Sigma)$, i.e. $A x_{G} \theta \subseteq T h_{\Sigma}\left(A x_{D}\right)$. Then one can easily observe that the following relations (i) - (iv) hold:
(i) $T h_{\Sigma}\left(A x_{G} \theta\right) \subseteq T h_{\Sigma}\left(A x_{D}\right)$
(ii) $T h_{\Sigma_{\theta}}\left(A x_{G} \cup D e f_{\theta}\right) \cap \operatorname{Sen}(\Sigma)=T h_{\Sigma}\left(A x_{G} \theta\right)$
(iii) $T h_{\Sigma_{\theta}}\left(A x_{G} \cup D e f_{\theta}\right) \cap \operatorname{Sen}(\Sigma) \subseteq T h_{\Sigma}\left(A x_{D}\right)$
(iv) $T h_{\Sigma_{\theta}}\left(A x_{G} \cup D e f_{\theta} \cup A x_{D}\right) \cap \operatorname{Sen}(\Sigma)=T h_{\Sigma}\left(A x_{D}\right)$

The first formula just states that all covered formulas are domain formulas, a necessary condition to call $A x_{G}$

\footnotetext{
${ }^{6}$ The $\Sigma_{\theta}$-formulas $D e f_{\theta}$ are naturally induced by the theory of "restricted higher-order anti-unification" as described in (SKKG09).
}

a generalization of the domain. The second formula describes the covered part of the domain theory as a (subset of) the closure under semantic entailment of $A x_{G} \cup D e f_{\theta}$. The third formula is a trivial consequence from (i) and (ii). The fourth formula states that adding $A x_{G} \cup D e f_{\theta}$ does not change the set of entailed formulas in $\operatorname{Sen}(\Sigma)$. It is an immediate consequence of (iii).

Using the contravariant satisfaction constraint of the theory of institutions, we can characterize the corresponding model classes as dual statements of the above results as follows:

(i) $\operatorname{Mod}_{\Sigma}\left(A x_{G} \theta\right) \supseteq \operatorname{Mod}_{\Sigma}\left(A x_{D}\right)$

(ii) $\operatorname{Mod}_{\Sigma_{\theta}}\left(A x_{G} \cup D e f_{\theta}\right) \cong \operatorname{Mod}_{\Sigma}\left(A x_{G} \theta\right)$

(iii) $\operatorname{Mod}_{\Sigma_{\theta}}\left(A x_{G} \cup D e f_{\theta}\right) \hookleftarrow \operatorname{Mod}_{\Sigma}\left(A x_{D}\right)$ is injective.

(iv) $\operatorname{Mod}_{\Sigma_{\theta}}\left(A x_{G} \cup D e f_{\theta} \cup A x_{D}\right) \cong \operatorname{Mod}_{\Sigma}\left(A x_{D}\right)$

The crucial point is again, that we have no choice when interpreting the new symbols, as their value is determined by the defining equations from $D e f_{\theta}$.

\section{Describing Analogies}

We will now turn to analogies and their description in HDTP. The starting point is given by two domain axiomatizations $A x_{S}$ and $A x_{T}$ over signature $\Sigma_{S}$ and $\Sigma_{T}$, respectively. HDTP will then compute a set of generalized formulas $A x_{G}$ and substitutions $\sigma, \tau$ such that $T h_{\Sigma_{S}}\left(A x_{G} \sigma\right) \subseteq T h_{\Sigma_{S}}\left(A x_{S}\right)$ and $T h_{\Sigma_{T}}\left(A x_{G} \tau\right) \subseteq$ $T h_{\Sigma_{T}}\left(A x_{T}\right)$. Following the HDTP process described above we can construct signatures $\Sigma_{S, \sigma}$ and $\Sigma_{T, \tau}$ such that $A x_{G}$ are first-order formulas over these signatures. We set $\Sigma_{G}=\Sigma_{S, \sigma} \cap \Sigma_{T, \tau}$ and conclude that $A x_{G} \subset \operatorname{Sen}\left(\Sigma_{G}\right)$.

For the following discussion we introduce some abbreviations:

$$
\Sigma_{S}^{+}:=\Sigma_{S} \cup \Sigma_{G} \quad \text { and } \quad \Sigma_{T}^{+}:=\Sigma_{T} \cup \Sigma_{G}
$$

Then the sets of equations $D e f_{\sigma}$ and $D e f_{\tau}$ are formulas over $\Sigma_{S}^{+}$and $\Sigma_{T}^{+}$, respectively. Furthermore, we will set $A x_{S}^{+}:=A x_{S} \cup D e f_{\sigma}$ and $A x_{T}^{+}:=A x_{T} \cup D e f_{\tau}$. We have the following inclusion relations between signatures:

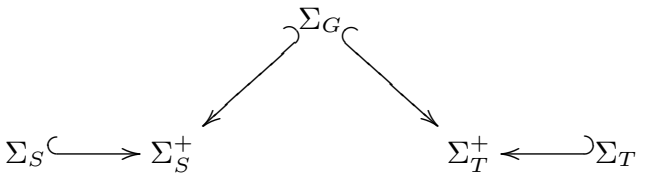

These signature inclusions induce inclusions on the syntactic level (notice that we work in the institution FOL):

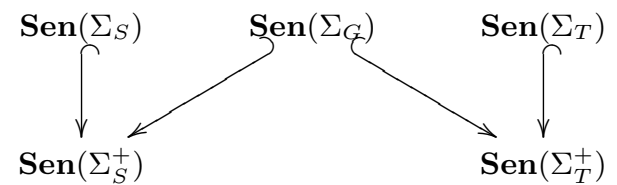

Dually the above signature inclusions induce the inverse inclusions on the semantic level: 


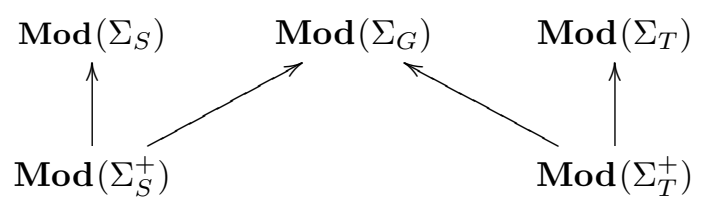

We will now examine the subsets of these sets, that are associated with the domain theories. Compare Figure 1 for these subset relations. The claim that $i_{S}$ and $i_{T}$ are inclusions, follows from

$$
\left(A x_{S}^{+} \cup A x_{G}\right)^{* *}=\left(A x_{S}^{+}\right)^{* *}
$$

On the semantic level $\tilde{\mathrm{j}}_{S}$ and $\tilde{\mathrm{j}}_{T}$ are isomorphisms (even though $j_{S}$ and $j_{T}$ are not), while $\tilde{1}_{S}$ and $\tilde{\mathrm{i}}_{T}$ are in general neither injective nor surjective.

\section{Analogical Relation}

Given the considerations so far, we can define the following mappings (cf. Figure 1):

$$
\begin{gathered}
m_{S}:\left(A x_{S}\right)^{*} \rightarrow\left(A x_{G}\right)^{*} \quad \text { and } \\
m_{T}:\left(A x_{T}\right)^{*} \rightarrow\left(A x_{G}\right)^{*}
\end{gathered}
$$

by $m_{S}=\tilde{\mathrm{i}}_{S} \circ \tilde{\mathrm{j}}_{S}^{-1}$ and $m_{T}=\tilde{\mathrm{i}}_{T} \circ \tilde{\mathrm{j}}_{T}^{-1}$. Given models $M_{S} \in\left(A x_{S}\right)$ and $M_{T} \in\left(A x_{T}\right)$, for every symbol $x \in \Sigma_{G}$ we get two interpretations $m_{S}\left(M_{S}\right)(x) \in U_{S}$ and $m_{T}\left(M_{T}\right)(x) \in U_{T}$. Associating those elements establishes the analogical relation. Briefly, this is the idea to relate $M_{S}(x \sigma) \sim M_{T}(x \tau)$ which corresponds to the relation $x \sigma \sim x \tau$ on the syntactic level. Clearly, it is possible to extend this association to terms and even formulas.

\section{Example: Heat Flow}

It might be worth to spell out these ideas in an example. We have chosen the heat flow analogy, which models the creation of new concepts "heat" and "heat flow" by analogical inference, given a model of water flow as the source domain. In the context of HDTP, this analogy seems to be especially interesting as it makes some complex mappings necessary. For a detailed discussion of the computation compare (SKKG09)).

We will follow (GB92) and describe signatures by two sets: function symbols and predicate symbols, both annotated with arity. So we start with the following domain signatures and axiomatizations:

$$
\begin{aligned}
& \Sigma_{S}=\{\quad\{\text { height } / 2 \text {, in } / 2, \text { water } / 0, \text { beaker } / 0 \text { vial } / 0, \\
& \left.\left.t_{1} / 0, t_{2} / 0\right\},\{>/ 2\}\right\} \\
& A x_{S}=\left\{\text { height(water in beaker, } t_{1}\right)> \\
& \text { height (water in vial, } \left.t_{1}\right) \rightarrow\left(t_{1}>t_{2}\right) \\
& \left.\wedge \text { height (water in beaker, } t_{1}\right)> \\
& \text { height (water in beaker, } t_{2} \text { ) } \\
& \left.\wedge \text { height (water in vial, } t_{2}\right)> \\
& \text { height(water in vial, } \left.t_{1}\right) \text {, } \\
& \ldots\}
\end{aligned}
$$

The signatures $\Sigma_{S}$ of the source domain provides the vocabulary to describe the flow of water in two connected vessels, a beaker and a vial. Due to space restriction we have only depicted one particular fact from the axiomatization: if the water level in the beaker is higher than the water level in the vial at a given time point $t_{1}$, then for every subsequent observation the water level will decline in the beaker while it will increase in the vial. For the target domain we provide the following axiomatization:

$$
\begin{gathered}
\Sigma_{T}=\left\{\begin{array}{l}
\{\text { temp } / 2, \text { in } / 2, \text { coffee } / 0, \text { cup } / 0, \text { cube } / 0 \\
\left.\left.t_{1} / 0, t_{2} / 0\right\},\{>/ 2\}\right\}
\end{array}\right. \\
A x_{T}=\left\{\begin{array}{l}
\text { temp }\left(\text { coffee in cup }, t_{1}\right)>\text { temp }\left(\text { cube }, t_{1}\right) \\
\rightarrow\left(t_{1}>t_{2}\right) \\
\wedge \text { temp }\left(\text { coffee in cup }, t_{1}\right)> \\
\text { temp }\left(\text { coffee in cup }, t_{2}\right) \\
\wedge \text { temp }\left(\text { cube }, t_{2}\right)>\text { temp }\left(\text { cube }, t_{1}\right), \\
\ldots\}
\end{array}\right.
\end{gathered}
$$

The vocabulary provides elements to describe a situation in which a cup of hot coffee, when connected with an cold ice cube, will cool down, while the ice warms up. From this axiomatization, HDTP will compute the set of generalized formulas:

$$
\begin{aligned}
A x_{G}=\left\{\begin{array}{l}
E \\
\rightarrow
\end{array}\right. & \left(t_{1}>t_{1}\right)>E\left(Y, t_{1}\right) \\
& \wedge E\left(X, t_{1}\right)>E\left(X, t_{2}\right) \\
& \wedge E\left(Y, t_{2}\right)>E\left(Y, t_{1}\right), \\
& \ldots\}
\end{aligned}
$$

and substitutions

$$
\begin{aligned}
\sigma & =\{E / \lambda u, \text { v.height }(\text { water in } u, v), X / \text { beaker }, Y / \text { vial }\} \\
\tau & =\{E / \lambda u, \text { v.temp }(u, v), X / \text { coffee in cup }, Y / \text { cube }\}
\end{aligned}
$$

The formulas in $A x_{G}$ are (higher-order) formulas over the signature

$$
\Sigma_{S} \cap \Sigma_{T}=\left\{\left\{t_{1} / 0, t_{2} / 0\right\},\{>/ 2\}\right\}
$$

with generalization variables $E, X, Y$. Now, interpreting these variables (that occur in the domain substitutions) as new symbols, we get an extended signature:

$$
\Sigma_{G}=\left\{\left\{E / 2, X / 0, Y / 0, t_{1} / 0, t_{2} / 0\right\},\{>/ 2\}\right\}
$$

The defining equations $D e f_{\sigma}$ and $D e f_{\tau}$ are constructed as sets of formulas over the signatures $\Sigma_{G}^{+} \cup \Sigma_{S}$ and $\Sigma_{G}^{+} \cup \Sigma_{T}$, respectively: 


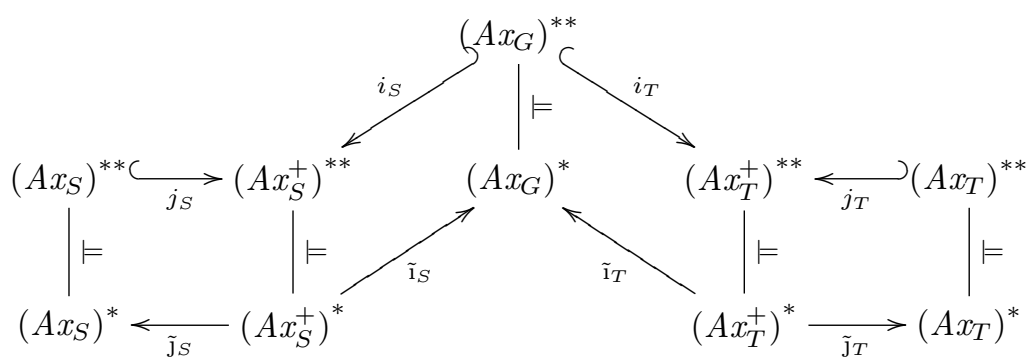

Figure 1: Subset relations that are associated with the domain theories.

$$
\begin{gathered}
\text { Def } f_{\sigma}=\left\{\begin{array}{l}
\forall u, v: E(u, v)=\text { height }(\text { water in } u, v), \\
X=\text { beaker, } Y=\text { vial }\}
\end{array}\right. \\
D e f_{\tau}=\left\{\begin{array}{l}
\forall u, v: E(u, v)=\text { temp }(u, v), \\
X=\text { coffee in cup }, Y=\text { cube }\}
\end{array}\right.
\end{gathered}
$$

As can be seen from these formulas, the complex expression height (water in $u, v$ ) on the source side corresponds to the less complex term $\operatorname{temp}(u, v)$ on the target side, while the complex expression coffee in cup on the target corresponds to the constant beaker on the source.

\section{Conclusions}

In this paper, we propose that analogy making can be used for various applications in the AGI context. We sketched the framework HDTP for analogy making that is intended to compute a generalized theory of given input source and target domains. The main contribution of this paper is a new institution-based formal semantics of the established analogical relation together with the model theory of the computed generalized theory. A classical example of analogy making is also roughly sketched. Future work will be a complete specification of the model theory of analogical relations, a representative set of worked out examples, and the discussion of potential alternatives to the present approach.

\section{References}

R. Diaconescu. Institution-Independent Model Theory. Studies in Universal Logic. Birkhäuser, 2008.

M. Dastani, B. Indurkhya, and R. Scha. An algebraic approach to modeling analogical projection in pattern perception. Journal of Experimental and Theoretical Artificial Intelligence, 15(4):489-511, 2003.

B. Falkenhainer, K. Forbus, and D. Gentner. The structure-mapping engine: Algorithm and examples. Artificial Intelligence, 41(1):1-63, 1989.

J. Goguen and R. Burstall. Institutions: Abstract model theory for specification and programming. Journal of the ACM, 39(1):95-146, 1992.

D. Gentner, K. Holyoak, and B. Kokinov, editors. The Analogical Mind. Perspectives from Cognitive Science. MIT Press, Cambridge, Mass, 2001.
H. Gust, U. Krumnack, K.-U. Kühnberger, and A. Schwering. An approach to the semantics of analogical relations. In Proceedings of the EuroCogSci 2007, 2007.

H. Gust, K.-U. Kühnberger, and U. Schmid. Metaphors and heuristic-driven theory projection. Theoretical Computer Science, 354(1):98-117, 2006.

H. Gust, U. Krumnack, A. Schwering, and K.-U. Kühnberger. The role of logic in AGI systems: Towards a Lingua Franca for general intelligence. In Proceedings of the Second Conference of Artificial General Intelligence, 2009.

B. Goertzel and C. Pennachin. Preface. In B. Goertzel and C. Pennachin, editors, Artificial General Intelligence. Springer, 2007.

J. Hummel and K. Holyoak. LISA: A computational model of analogical inference and schema induction. In Proceedings of 16th Meeting of the Cognitive Science Society, 1996.

D. Hofstadter. Fluid Concepts and Creative Analogies. Basic Books, 1995.

B. Indurkhya. Metaphor and Cognition. Kluwer Academic Publishers, Dordrecht, 1992.

B. Kokinov, K. Holyoak, and D. Gentner, editors. Proceedings of the Second International Analogy Conference - Analogy 09, New Frontiers in Analogy Research. NBU Press, 2009.

B. Kokinov and A. Petrov. Integrating memory and reasoning in analogy-making: The AMBR model. In D. Gentner, K. Holyoak, and B. Kokinov, editors, The Analogical Mind. Perspectives from Cognitive Science, pages 59-124. MIT Press, 2001.

G. Plotkin. A note on inductive generalization. $M a-$ chine Intelligence, 5:153-163, 1970.

A. Schwering, K.-U. Kühnberger, and B. Kokinov, editors. Special Issue on Analogies - Integrating Cognitive Abilities, volume 10 of Cognitive Systems Research. Elsevier, 2009.

A. Schwering, U. Krumnack, K.-U. Kühnberger, and H. Gust. Syntactic principles of heuristic-driven theory projection. Cognitive Systems Research, 10(3):251269, 2009. 